\title{
The Dubrava Model-A Novel Approach in Treating Acutely Neurotraumatized Patients in Rural Areas: A Proposal for Management
}

\author{
Darko Orešković ${ }^{1}$ Marina Raguž ${ }^{1}$ Fadi Almahariq1 ${ }^{1}$ Domagoj Dlaka' Dominik Romić \\ Petar Marčinković ${ }^{1}$ Anđelo Kaštelančić ${ }^{1}$ Darko Chudy ${ }^{1}$ \\ ${ }^{1}$ Department of Neurosurgery, University Hospital Dubrava, Zagreb, \\ Address for correspondence Marina Raguž, MD, PhD, Department \\ Croatia \\ of Neurosurgery, University Hospital Dubrava, Avenija Gojka Suska 6, \\ Zagreb HR-10000, Croatia (e-mail: marinaraguz@gmail.com).
}

J Neurosci Rural Pract 2019;10:446-451

\begin{abstract}
Introduction Neurotrauma is one of the leading causes of death and disabilities nowadays and represents one of the largest socioeconomic problems in rich countries, as well as developing ones. A satisfying, medically viable, and cost-effective model of managing acutely neurotraumatized patients, especially ones who come from distant and/or rural areas, has yet to be found. Patient outcome after acute neurotrauma depends on many factors of which the possibility of urgent treatment by an experienced specialist team has a crucial role. Here, we present our own way of managing acutely neurotraumatized patients from distant places which is unique in Croatia, the Dubrava model.

Methods We present our 5-year experience cooperating with general hospitals in four neighboring cities (Čakovec, Bjelovar, Sisak, and Koprivnica) in managing, operating, and taking care of acutely neurotraumatized patients.

Results More than 300 surgeries have been performed in these hospitals through the Dubrava model. Our experience so far provides encouraging results that this system could also be successfully implemented in other institutions. Furthermore, we recorded an increased number of surgeries each year, as well as a good mutual cooperation with the local general hospitals.

Discussion This trauma managing model is one of a kind in Croatia. We argue that it is not only better for the patients, providing them with better chances of survival, and disability-free recovery, but is also far superior in many ways to the dominant and

Keywords

- acute neurotrauma

- the Dubrava model

- rural areas

currently prevalent way of treating these patients in other parts of Croatia.

Conclusion The Dubrava model of treating patients in rural and distant areas is a reliable and proven model with many benefits and as such its implementation should be considered in other institutions as well.
\end{abstract}

\section{Introduction}

Traumatic brain injuries (TBIs) or neurotraumas are one of the leading causes of death and disability and are an enormous socioeconomic and health care problem around the world. ${ }^{1-3}$ Conservative estimates are that in the European Union (EU), for example, the number of hospitalizations and deaths after brain trauma is between 200 and 600 in 100,000 people annually. ${ }^{2,3}$ Brain traumas are also the biggest cause of years of population disability and are responsible for up to half of deaths after trauma. ${ }^{2,4}$ Data and statistics regarding the exact number of patients, types of injuries, and so on, are unfortunately hard to collect and analyze. This is due not only to the fact that, especially in developing nations, there 
are few (if any) institutions responsible for data collection, but also because there are very few clear, obligatory, and precise protocols of collecting data and its analysis. ${ }^{2}$ However, even not taking into account the disabilities and death after neurotrauma, it has been estimated that only in the EU live almost 8 million people who suffer from some sort of relatively milder consequences of TBIs, such as attention deficit disorder, depression, impulsivity, difficulties in making decisions, or aggression. ${ }^{5}$ All of this shows what an enormous burden neurotraumas are today, not only for affected individuals but their families and the society as a whole.

Current guidelines for taking care of acutely neurotraumatized patients are mostly developed based on research conducted in some of the wealthiest countries in the world. ${ }^{6}$ It has been recognized, however, that these guidelines are not sufficient for every country and that they do not bring the same or even similar results in different countries. ${ }^{6,7}$ There is a certain consensus in literature today that one should try to take into consideration various medical, economic, geographic, and other unique aspects of each country when devising and organizing a system of taking care of these patients. ${ }^{6,7}$

In Croatia, treatment of acutely neurotraumatized patients, who come from distant, rural areas, has been resolved in a way which is similar to the one implemented by many Western countries. In this system, neurotraumatized patients are first transported to a local hospital with the retrieval team. From here, the patients' medical history, status, and images are being sent to a neurosurgeon at a bigger center through telemedicine system, after which the surgeon makes an indication to perform emergency surgery. If this is the case, patients are being transported yet again to these bigger centers with neurosurgery departments where they can be operated on. After surgery, the patients usually continue their recovery at these neurosurgery departments without returning to the initial hospital. This was indeed the protocol for patient treatment at our institution as well. Neurotraumatized patients from the north of Croatia, more specifically from the towns of Čakovec, Bjelovar, Sisak, and Koprivnica, were transported first to the general hospitals in these towns; after which, if emergency surgery was needed, they were transported to our institution where they were operated on and continued with their recovery and rehabilitation.

This is the prevalent and most common way of treating patients with TBIs, since neurosurgery being one of the most specialized branches of medicine, and also is both medically and economically viable to exist in big, central hospitals. Also, surgeons' experience has been shown to be one of the most important factors in the patient outcome, neurosurgery, ${ }^{8-10}$ as well as other branches of surgery. ${ }^{10-12}$ Smaller hospitals in Croatia, as in other countries, must therefore transfer acutely neurotraumatized patients, regardless of how bad their condition might be, to distant places, wasting precious time and risking transporting patients for $50,100,200$, or sometimes even more $\mathrm{km}$ to bigger hospitals with neurosurgery departments.

\section{Methods}

However, 5 years ago, a novel and unique approach has been developed, the so-called Dubrava model. This model has, in our opinion, many benefits compared with the traditional system which makes it a notable improvement to the current protocol used in other parts of Croatia. Ever since the implementation of this model, acutely neurotraumatized patients from these hospitals are not being transported to University Hospital Dubrava (UH Dubrava) anymore to be operated on, instead a neurosurgeon from UH Dubrava used to go to one of these hospitals to perform the surgery. This approach, again, is a unique system in Croatia and its implementation is discussed next.

The beginning of treating the patient is the same, as it was before the implementation of the model. Acutely neurotraumatized patients are transported to the local general hospital with the retrieval team. The patient's medical history, status, laboratory results, and all other relevant information are taken at the emergency departments as usual and according to the current guidelines. The attending physician then contacts the on-call neurosurgeon who is available 24 hours a day at the UH Dubrava and sends him the relevant medical documentation and images through the telemedicine system. The neurosurgeon then indicates an appropriate course of further treatment. This may simply involve consultation about the medication and further radiologic and neurologic examinations. However, in the event that emergency surgery is indicated, the course of action which is taken by our institution differs from other neurosurgery departments in Croatia.

If emergency surgery is needed, the on-call neurosurgeon at the UH Dubrava informs another available neurosurgeon about all the necessary information regarding the patient (medical history, status, and radiographic images). The available neurosurgeon then goes to perform the surgery in aforementioned local general hospital. He drives with his personal vehicle to the town in question. While this neurosurgeon is traveling, the one at the hospital informs the physicians at the local hospital of the plan of treatment, gives detailed instructions concerning the patient's preoperative preparation, medication, possible further laboratory tests, and so on. This assures that the patient is ready and prepared for surgery by the time the neurosurgeon arrives without losing valuable time.

Of importance, besides the on-call neurosurgeon who is in the UH Dubrava, the other neurosurgeon is also available for travel and able to perform emergency surgery 24 hours a day. In the case, this neurosurgeon goes to another town to perform the surgery, another neurosurgeon takes his place as the available one. The list and order of on-call and available neurosurgeons is of course made at the beginning of each month. However, we must note that in our experience, it is rarely the case that two or more neurosurgeons are needed to perform these surgeries at the same time.

Hence, when the neurosurgeon arrives at the local hospital, patient and operating-room (OR) team are ready and prepared for surgery. The ORs in these hospitals are equipped with all the instruments and equipment needed for this type of emergency surgery (Mayfield clamps, craniotomy sets, etc.). After surgery, the patient is usually admitted to the intensive care unit (ICU). All of the four general hospitals with whom we collaborate have experienced and capable 
ICU teams, as well as equipment, and facilities adequate for treating even the most difficult and severe cases of TBI, for both postsurgical and nonsurgical patients. After their treatment in the ICU, the patients are usually admitted to trauma or surgery departments in these hospitals to continue their treatment and rehabilitation. Sometimes, especially if the patient was presented to the neurology emergency room on arrival, they will be transferred to the neurology department instead. Furthermore, each one of the four general hospitals has a physiotherapy and rehabilitation department with resident physicians, as well as physiotherapists, to aid and assist the recovery of these patients for the duration of their stay at the hospital. Worth noting is that, at each step of the patient's recovery, the physicians at these hospitals have direct contact with our neurosurgical team, as well as the operating physician, 24 hours a day. This is usually done through the telemedicine system (for computed tomography scans, neurologic status, laboratory results), as well as direct phone calls. The operating neurosurgeon will give detailed instructions not only regarding the postoperative patient management, medication, required radiographic imaging, and wound care but also regarding future controls and recommendations after discharge. Indeed, after discharge, the patients are usually scheduled for control clinic visits with their operating neurosurgeon at the UH Dubrava. If the patient was not operated on and was treated conservatively, their control is also scheduled and arranged at our hospital with the neurosurgeon who was on-call on the day of the admission to the local hospital and who provided instructions regarding patient care.

Worth noting, here is the geography of this part of Croatia. The distances of aforementioned towns to Zagreb are as follows. The town of Sisak is the closest one and is approximately $60 \mathrm{~km}$ away from Zagreb, Bjelovar, is around $90 \mathrm{~km}$, while both Koprivnica and Čakovec are around $100 \mathrm{~km}$ from Zagreb. Of note, Croatia has a very tourism-oriented economy with many tourists coming from central Europe (the north of Croatia) and traveling to the seaside in the south. This is fortunate since high-quality highways and freeways have therefore been built throughout the country, including this part of Croatia. This, in turn, means that all of these distances can be traveled with speed, comfort, and ease. Indeed, even to the most distant towns (Koprivnica and Čakovec), it takes little more than an hour for a neurosurgeon in a personal vehicle to arrive from his home to the hospital.

\section{Results}

In the past 5 years, over 320 emergency surgeries have been made in these four general hospitals. The number of surgeries was roughly the same in all of them (around 80). By far, the most prevalent indication for surgery was subdural hematomas with 170 patients operated on due to, primarily, this diagnosis. Of these, 117 patients presented with an acute subdural hematoma, while 53 patients were operated on because of a chronic or subacute hematoma. Patients with epidural hematomas were operated on 30 times, while there were 49 operations on patients with intracerebral hemorrhage. Severe fracture of the neurocranium was a primary indication for surgery in 43 patients. The operations for 28 patients were labeled as "other," meaning that they could not be described into these categories. Worth noting is that in addition to these, there were also 21 reoperations, due to postoperative hemorrhage, newly developed hematomas, and so on.

\section{Discussion}

Having explained our model, we will discuss the benefits of our system, as we see them, and why it was implemented in the first place. The need for this approach came for several reasons.

The most important ones are of course medical. We feel that the literature is quite clear by now that patient transfer should be minimized, especially ones with such serious injuries. This is due to many possible complications and risks, many of which are of course minimized by the expertise of the transporting staff, but are present, nevertheless. Indeed, it has been shown that the transport of patients even within the same hospital (for diagnostics and treatment) carries certain risks and possibilities for complications. ${ }^{13}$ Transferring these patients to other hospitals, and especially to hospitals in different towns, multiply these risks, which has also been shown throughout the years with various studies (recent data $^{13-15,20}$ and older papers on the topic ${ }^{16,19,21,22}$ ). In Great Britain, for example, acutely neurotraumatized patients who were being transported from other hospitals had a greater risk of developing a wide variety of complications from relatively more benign ones, such as hyper- and hypotension, bradi- and tachycardia, hypo- and hypertension, heart arrhythmias, and hypoxias, all the way to severe ones, such as pulmonary embolism, pneumothorax, various forms of hemorrhage, elevated intracranial pressure, and death. ${ }^{13}$ Other studies mentioned that transporting patients not only include a higher risk of movement, disconnection, or breaking of various tubes and catheters but also of difficult monitoring and communication due to the noise and vibrations present during transport. ${ }^{23}$ Many other nonmedical problems are also present, such as troubles with functioning or malfunction of various equipment, as well as complications with the transport itself, all of which are of course risks always present in transporting patients. ${ }^{23}$ Finally, it is worth mentioning that many acutely traumatized patients due to the nature of their injuries are not even able to handle being transported to another hospital or town. It is thus already well-established that patient transfer (both inter- and intrahospital) should be minimized (further reading and more in-depth analyses of different types of patient transfer and management ${ }^{24-31}$ ). Again, the risk of many of these complications can be minimized by the expertise of the transporting team, but these risks are in fact present and pose a serious risk to the patient's well-being. Even though the retrieval teams in our country are capable of transporting severely injured patients across large distances (even the ones requiring intense monitoring and intubation), we feel these inherit risks of transport are avoided using our model. 
Other than an increased number of complications during transport, research has shown that patients who were transferred between hospitals were more ill (they scored higher values in "Acute Physiology" as well as "Chronic Health Evaluation III Score"14,15). Furthermore, it has been shown that these patients had a higher mortality rate and a longer stay in hospitals compared with the ones who were not transferred. ${ }^{14,15}$ More resources were also spent on these patients. In the United States, for example, on average, 9,000\$ more were spent on a patient who was transferred to another hospital. ${ }^{14}$ On the other hand, a meta-analysis conducted in 2011 couldn't conclude that interhospital transport carries a higher incidence of complications, although the authors found it likely that the reported risk had been underestimated. ${ }^{15}$

Beside these strictly medical reasons, there are many other socioeconomic ones why we consider the Dubrava model to be the best one in Croatia in treating acutely neurotraumatized patients.

The first one being that unlike the Dubrava model, the classical way of managing acutely neurotraumatized patients leads to centralization and clogging of the system. In Croatia's health care system, where the waiting lists for diagnostics and treatment are quite long, we see the Dubrava model as an ideal way to decentralize and help to develop smaller hospitals. Indeed, besides patient safety, this was one of our main reasons for implementing our model in the first place. Before, our ORs, ICUs, and department facilities were being used to the maximum with many TBI patients. By the sheer nature of their injury, these patients require a long time to recover and rehabilitate. Since all of our patients who undergo elective neurosurgery involving a craniotomy spend the first postoperative night in the ICU, this meant that our regular elective operating program was often being delayed significantly if not cancelled until the ICU beds were made available. There were emergency surgeries almost every night at our hospital which was of course very difficult and exhausting. With our model, this problem was solved not only effectively but also in a way that the quality of health care in smaller hospitals did not diminish. With the Dubrava model, many more of our patients can now be admitted more rapidly and operated on sooner. Of these patients, we would like to primarily point out those with brain glial tumors whose overall survival is directly correlated with the speed and efficacy at which they can be operated on and then referred to further oncological treatment after surgery. The Dubrava model also justifies the existence and founding of smaller hospitals, their staff, their ORs, and ICUs, and provides them to do exactly what they were made for and which is to take care of patients.

The second reason is that we feel that transferring patients to another city loses precious time. Even after the patient has been transferred to a bigger hospital which is capable of managing his condition, there is an amount of time required to prepare the patient, the OR, the OR staff, and so on. This waste of time can literally mean the difference between life and death for these patients. We feel that with our model, one loses far less time. Most notably because after the indication for emergency surgery has been made, the patient is immediately being prepared for surgery. Therefore, no time is being wasted for preparation; everything is being done, while the surgeon is on his way. Furthermore, our experience shows that one can travel faster with a personal vehicle than with an ambulance car considering that Croatia, as mentioned earlier, is very well connected with excellent roads. An ambulance can of course travel much faster within the city, especially during traffic hours, but in our experience, this has never been a problem. We acknowledge that more data are needed, primarily a comparison between different times of arrival and beginnings of operations, outcomes, costs, and so on, both before and after the implementation of the model. However, due to the fact that these data have to be retrieved from institutions which are not our own, collecting reliable data has unfortunately proven to be quite challenging. Thus, we still do not have reliable data on such a big number of surgeries. However, we feel that the logical arguments we are presenting, alongside the fact that this model has been satisfactory for quite some time now, not only to employees of our institution but also the ones at the local general hospitals, will be enough for many hospitals and neurosurgery departments to seriously consider implementing a model similar to ours.

The third reason why we advocate the Dubrava model is that transporting patients is expensive. Transporting acutely traumatized patients to different hospitals and cities requires a great logistic and economic expense. According to our data, transporting a patient around $200 \mathrm{~km}$ costs around 1,500\$. This high price is needed since educated and experienced personnel are imperative in transporting such patients. The arrival of just one surgeon is by far less expensive.

The fourth reason is that young doctors from smaller hospitals, who do not have many opportunities to see acutely neurotraumatized patients, let alone have the chance to operate on them, with the Dubrava model gain experience from assisting and working with some of the best and most experienced neurosurgeons in Croatia. The first-hand experience received this way is of course invaluable in the lasting education of a young surgeon. An interesting comparison can be made between Croatia and Australia in this regard. Australia, having such a vast territory, has considerable experience in treating patients with acute neurotraumas in distant and remote areas. Australasian neurosurgical society has thus issued guidelines ${ }^{32}$ to local surgeons in which they can perform emergency neurosurgery under the guidance and agreement from a neurosurgeon and if they are more than 2-hour away from a neurosurgery department. ${ }^{33}$ The guidelines also include the possibility of a neurosurgeon traveling with the retrieval team to assist the local surgeon which is something quite similar to our model. Furthermore, transit complications whether by helicopter or fixed wing aircraft have been reported to be uncommon. ${ }^{34,35}$ Our department in fact has a similar agreement with the local surgeons. Since they already have more than 5 years of experience assisting in neurosurgical operations, if ever the need arises for such a rapid treatment where the patient simply cannot wait for the neurosurgeon to arrive from Zagreb (for example, patients with epidural hematomas with such a sharp decline in their neurologic status), these surgeons, as well as the OR staffs, 
are more than capable of positioning the patient, starting the procedure, and performing the emergency craniotomy. This also is something which simply could not have been possible before. However, we must note that, fortunately, this course of action has not been needed, to date.

The fifth and final reason is one of the human nature. The fact remains that when patients are admitted to a big, distant hospital, they are far away from their family. Of course, it is much easier for people to travel a couple of kilometers to a hospital than to travel more than a hundred kilometers each day. And the vicinity of injured patients to their families, as well as the possibility of their frequent visits, is beneficial for everyone involved.

\section{Conclusion}

All of the above are the reasons why we feel that the Dubrava model of taking care of acutely neurotraumatized patients is by far superior to the classical and so-far dominant. With this model, an area of around $500 \mathrm{~km}^{2}$ is being covered, along with more than 200,000 people living just in the cities and towns where the smaller hospitals are located. This number is of course much larger since there are a lot of people living in nearby villages who gravitate toward these towns. One could say that almost the entire northern part of Croatia is covered with our model which brought these people access to some of the best and most experienced neurosurgeons in Croatia, not diminishing the quality of health care in smaller hospitals, saving money for the health care system, and helping the development of smaller, rural areas. We encourage other tertiary centers, as well as smaller hospitals, both in Croatia and abroad, to publish their suggestions and critiques of our model, all for the purpose of advancing and improving the management and survival of our acutely neurotraumatized patients.

\section{Conflict of Interest}

None declared.

\section{References}

1 Alves ÓL, Bullock R, Excitotoxic damage in traumatic brain injury. In: Clark RSB, Kochanek P, eds. Brain Injury: Molecular and Cellular Biology of Critical Care Medicine. Vol. 2, 1st ed. Boston, MA: Springer; 2001:1-36

2 Rubiano AM, Carney N, Chesnut R, Puyana JC. Global neurotrauma research challenges and opportunities. Nature 2015;527(7578):S193-S197

3 Maas AI, Stocchetti N, Bullock R. Moderate and severe traumatic brain injury in adults. Lancet Neurol 2008;7(8):728-741

4 Valadka $A B$, Injury to the cranium. In: Moore EE, Feliciano DV, Mattox KL, editors. Trauma. 1st ed. New York: McGraw-Hill Medical Publication; 2004:385-406

5 Hofman K, Primack A, Keusch G, Hrynkow S. Addressing the growing burden of trauma and injury in low- and middle-income countries. Am J Public Health 2005;95(1):13-17

6 Maas AI, Menon DK, Lingsma HF, Pineda JA, Sandel ME, Manley GT. Re-orientation of clinical research in traumatic brain injury: report of an international workshop on comparative effectiveness research. J Neurotrauma 2012;29(1):32-46
7 Maas AI, Roozenbeek B, Manley GT. Clinical trials in traumatic brain injury: past experience and current developments. Neurotherapeutics 2010;7(1):115-126

8 Lawton MT, Du R. Effect of the neurosurgeon's surgical experience on outcomes from intraoperative aneurysmal rupture. Neurosurgery 2005;57(1):9-15, discussion 9-15

9 Torné R, Rodríguez-Hernández A, Lawton MT. Intraoperative arteriovenous malformation rupture: causes, management techniques, outcomes, and the effect of neurosurgeon experience. Neurosurg Focus 2014;37(3):E12

10 Schmidt CM, Turrini O, Parikh P, et al. Effect of hospital volume, surgeon experience, and surgeon volume on patient outcomes after pancreaticoduodenectomy: a single-institution experience. Arch Surg 2010;145(7):634-640

11 Sosa JA, Bowman HM, Tielsch JM, Powe NR, Gordon TA, Udelsman R. The importance of surgeon experience for clinical and economic outcomes from thyroidectomy. Ann Surg 1998;228(3):320-330

12 Cahill PJ, Pahys JM, Asghar J, et al. The effect of surgeon experience on outcomes of surgery for adolescent idiopathic scoliosis. J Bone Joint Surg Am 2014;96(16):1333-1339

13 Blakeman TC, Branson RD. Inter- and intra-hospital transport of the critically ill. Respir Care 2013;58(6):1008-1023

14 Golestanian E, Scruggs JE, Gangnon RE, Mak RP, Wood KE. Effect of interhospital transfer on resource utilization and outcomes at a tertiary care referral center. Crit Care Med 2007;35(6):1470-1476

15 Hill AD, Fowler RA, Nathens AB. Impact of interhospital transfer on outcomes for trauma patients: a systematic review. J Trauma 2011;71(6):1885-1900

16 Braman SS, Dunn SM, Amico CA, Millman RP. Complications of intrahospital transport in critically ill patients. Ann Intern Med 1987;107(4):469-473

17 Andrews PJ, Piper IR, Dearden NM, Miller JD. Secondary insults during intrahospital transport of head-injured patients. Lancet 1990;335(8685):327-330

18 Smith I, Fleming S, Cernaianu A. Mishaps during transport from the intensive care unit. Crit Care Med 1990;18(3):278-281

19 Kanter RK, Tompkins JM. Adverse events during interhospital transport: physiologic deterioration associated with pretransport severity of illness. Pediatrics 1989;84(1):43-48

20 Gray A, Gill S, Airey M, Williams R. Descriptive epidemiology of adult critical care transfers from the emergency department. Emerg Med J 2003;20:242-246

21 Martin GD, Cogbill TH, Landercasper J, Strutt PJ. Prospective analysis of rural interhospital transfer of injured patients to a referral trauma center. J Trauma 1990;30(8):1014-1019

22 Valenzuela TD, Criss EA, Copass MK, Luna GK, Rice CL. Critical care air transportation of the severely injured: does long distance transport adversely affect survival? Ann Emerg Med 1990;19(2):169-172

23 Dunn MJ, Gwinnutt CL, Gray AJ. Critical care in the emergency department: patient transfer. Emerg Med J 2007;24(1):40-44

24 Park KD, Seo SJ, Oh CH, Kim SH, Cho JM. The effectiveness evaluation of helicopter ambulance transport among neurotrauma patients in Korea. J Korean Neurosurg Soc 2014;56(1):42-47

25 Bekelis K, Missios S, Mackenzie TA. Prehospital helicopter transport and survival of patients with traumatic brain injury. Ann Surg 2015;261(3):579-585

26 Gray A, Gill S, Airey M, Williams R. Descriptive epidemiology of adult critical care transfers from the emergency department. Emerg Med J 2003;20(3):242-246

27 Garwe T, Cowan LD, Neas B, Cathey T, Danford BC, Greenawalt P. Survival benefit of transfer to tertiary trauma centers for major trauma patients initially presenting to nontertiary trauma centers. Acad Emerg Med 2010;17(11):1223-1232 
28 Rivara FP, Koepsell TD, Wang J, Nathens A, Jurkovich GA, Mackenzie EJ. Outcomes of trauma patients after transfer to a level I trauma center. J Trauma 2008;64(6):1594-1599

29 Sampalis JS, Denis R, Fréchette P, Brown R, Fleiszer D, Mulder D. Direct transport to tertiary trauma centers versus transfer from lower level facilities: impact on mortality and morbidity among patients with major trauma. J Trauma 1997;43(2):288-295

30 Sampalis JS, Denis R, Lavoie A, et al. Trauma care regionalization: a process-outcome evaluation. J Trauma 1999;46(4):565-579

31 Clement RC, Carr BG, Kallan MJ, Wolff C, Reilly PM, Malhotra NR. Volume-outcome relationship in neurotrauma care. J Neurosurg 2013;118(3):687-693
32 The management of acute neurotrauma in rural and remote locations. Available from: http://www.nsa.org.au/Documents/ Information/Rural\%20and\%20Remote\%20Trauma.pdf. Accessed August 9, 2019

33 Simpson D, North B, Gilligan J, et al. Neurological injuries in South Australia: the influence of distance on management and outcome. Aust N Z J Surg 1984;54(1):29-35

34 Gilligan J, Reilly P, Pearce A, Taylor D. Management of acute traumatic intracranial haematoma in rural and remote areas of Australia. ANZ J Surg 2017;87(1,2):80-85

35 Gilligan J, From Accident site to the trauma center. In: Reilly PL, Bullock R, eds. Head Injury, Pathology and Management. 2nd ed. London: CRC Press; 2005:271-393 